
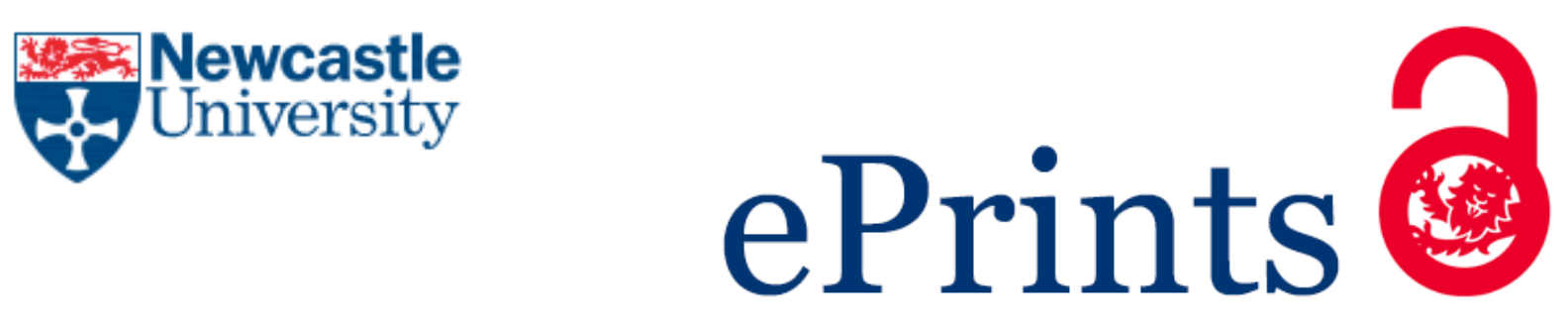

Baron S, Lazzarini N, Bacardit J.

Characterising the influence of rule-based knowledge representations in biological knowledge extraction from transcriptomics data. In: EvoApplications 2017: 20th European Conference on the Applications of Evolutionary Computation. 2017, Amsterdam: Springer.

Copyright:

The final publication is available at https://doi.org/10.1007/978-3-319-55849-3 9

DOI link to article:

https://doi.org/10.1007/978-3-319-55849-3 9

Date deposited:

$13 / 02 / 2017$

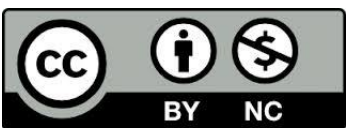

This work is licensed under a Creative Commons Attribution-NonCommercial 3.0 Unported License 


\title{
Characterising the influence of rule-based knowledge representations in biological knowledge extraction from transcriptomics data
}

\author{
Simon Baron, Nicola Lazzarini, and Jaume Bacardit \\ School of Computing Science \\ Newcastle University \\ Claremont Tower, Newcastle-upon-Tyne, NE1 7RU, UK \\ jaume.bacardit@newcastle.ac.uk
}

\begin{abstract}
Currently, there is a wealth of biotechnologies (e.g. sequencing, proteomics, lipidomics) able to generate a broad range of data types out of biological samples. However, the knowledge gained from such data sources is constrained by the limitations of the analytics techniques. The state-of-the-art machine learning algorithms are able to capture complex patterns with high prediction capacity. However, often it is very difficult if not impossible to extract human-understandable knowledge out of these patterns. In recent years evolutionary machine learning techniques have shown that they are competent methods for biological/biomedical data analytics. They are able to generate interpretable prediction models and, beyond just prediction models, they are able to extract useful knowledge in the form of biomarkers or biological networks.

The focus of this paper is to thoroughly characterise the impact that a core component of the evolutionary machine learning process, its knowledge representations, has in the process of extracting biologically-useful knowledge out of transcriptomics datasets. Using the FuNeL evolutionary machine learning-based network inference method, we evaluate several variants of rule knowledge representations on a range of transcriptomics datasets to quantify the volume and complementarity of the knowledge that each of them can extract. Overall we show that knowledge representations, often considered a minor detail, greatly impact on the downstream biological knowledge extraction process.
\end{abstract}

Keywords: evolutionary machine learning, rule knowledge representations, biological knowledge extraction

\section{Introduction}

Science and learning have always been built on the foundations of collecting and analysing data - in order to generate our knowledge in any field we must observe by collecting data and test by analysing that data against hypotheses. Currently, in bioinformatics, technological advances such as microarrays, high throughput sequencing and (perhaps most importantly) the sharing of information over the 
internet in collaborative databases, have greatly increased the amount of data we have been able to observe. Experimental technologies in transcriptomics and genomics are also designed to capture far more information than is required by the original researchers, which once again leads to a wealth of data easily available to anyone interested in studying the area further. In such an environment the focus has shifted to developing analytic tools and techniques that make the most of the data that is being collected. For this to happen we develop tools which are able to: (1) extract biological insight from raw data, (2) process large amounts of data relatively quickly and (3) produce outputs that are human readable.

Evolutionary machine learning techniques have been successfully applied for many years to analyse biological/biomedical data [16, 23, 4-6, 10, 22, 9, 24, 15, 13], in a variety of scenarios. In the majority of cases $[23,4-6,10,22,9,24,13]$ as supervised (classification) learning, but sometimes also applied to unsupervised learning in the form of association rules [16] or bi-clustering [15]. Moreover, they have been applied to a variety of biological data, such as transcriptomics $[16,6$, $10,15]$, SNPs [23, 24], proteomics [22], lipidomics [9], protein structure $[4,5]$ or clinical measurements [13]. Often, these methods are used for the core machine learning task of performing predictions, but in some cases also to extract knowledge from the data, as identifying and ranking important variables (biomarkers) $[23,24]$, generating minimal sets of biomarkers [22], or inferring networks of interactions from data $[16,6,23]$.

A method in this latter category, which we will use in this paper, is FuNeL [14]. FuNeL generates biological functional networks from a labelled (classification) dataset by mining the rule sets generated by the BioHEL evolutionary machine learning system [1]. In the generated networks the possible set of nodes are the attributes of the dataset (e.g. genes), and edges between two attributes are created if, in the rule sets generated by BioHEL, such pair of attributes is frequently observed. This network inference strategy is called co-prediction, given that attributes are connected not because they have similar values across samples (what the classic gene co-expression principle does) but because together these attributes make predictions on samples.

The co-prediction principle was originally used in [6], and what the authors observed in that work was that the generated network would be different depending on the core knowledge representation used in BioHEL. The standard BioHEL rules for continuous attributes are in the form of: "If attributeX $\in[2,4]$ and attributeY $\in[0,0.2]$ and $\ldots$ then predict class X". That is, for each relevant attribute a rule would specify a lower and an upper bound. If the representation was subtly changed so that rules would be in the shape "If attributeX $>2$ and attribute $Y>0$ and ... then predict class X" (where there are only lower bounds for each attribute), the networks generated by the co-prediction principle would be very different. This is a very interesting observation because it means that, by making very small changes to the core knowledge representations used in machine learning, the knowledge that can be discovered from a biological dataset can dramatically change. 
The aim of this paper is to systematically assess this phenomenon, using the combination of BioHEL+FuNeL. Four different types of rule predicates are explored: (1) AttX $\in$ [Lower,Upper], (2) AttX > Lower, (3) AttX $<$ Upper, and (4) AttX/AttY $>$ Threshold. We test both the case where BioHEL can construct rules using a single (fix) type of predicate and also when the genetic algorithm (GA) is free to discover the best combination of predicate types for each rule (mix). To assess the impact of these representations we build functional networks using FuNeL and then we assess the differences between such networks in relation to the set of nodes their contain but also the differences in the biological annotation of such nodes. To evaluate our methods we use 8 microarray datasets related to several different types of cancer.

The rest of the paper is structured as follows: Section 2 describes the algorithms involved in this work: BioHEL, FuNeL and the set of explored knowledge representations. Section 3 details our experimental validation protocol. Section 4 reports the results of our validation. Finally, section 5 summarises our findings and outlines future work.

\section{Methods}

\subsection{BioHEL}

BioHEL [1] is an evolutionary rule-based machine learning system originally designed to tackle large-scale datasets. BioHEL has been extensively used to analyse biological data of many different kinds: protein structure prediction [4, 5], transcriptomics [6,10], proteomics [22] or lipidomics [9]. For brevity, we are only describing here the aspects of BioHEL that are relevant to the present work.

Learning strategy BioHEL generates sets of classification rules using the iterative rule learning (IRL) principle, first introduced in the context of evolutionary machine learning in the SIA [25] system. In IRL, the rules that constitute a rule set are learnt sequentially. After each rule is learnt, the examples from the training set that it covers are removed in order to force the next rule to focus on a different part of the search space. In BioHEL a generational genetic algorithm is used to learn each of these rules. Hence, BioHEL evolves a population of individual rules. The IRL process generally finishes when all the training examples have been covered. In the case of BioHEL, in which rule sets have an explicit (and predefined) default rule, the process finishes then the GA cannot learn any rule that is better than the default rule.

Existing knowledge representation BioHEL uses a rule representation called Attribute List Knowledge Representation (ALKR) [1], which will be heavily refactored in this work. In ALKR, each rule has an embedded feature selection: each rule specifies the subset of attributes it uses, called the attribute list. This subset may differ across rules (GA individuals). In the case of datasets with continuous attributes, each rule encodes an hyper-rectangle: for each attribute 
within the list, an interval, with a lower bound and an upper bound, is specified. Any attribute not present in the list will be considered as irrelevant. A class is associated with each rule to predict the label of each sample matched by such rule. Each rule in the initial GA population will randomly pick a subset of attributes (a parameter of the system specifies how many). To discover the right attributes for each rule, the GA cycle is extended with two operators that probabilistically add (specialise) or remove (generalise) attributes from the rule.

Fitness function The fitness function of BioHEL is designed to strike a balance between accuracy (rules should not make mistakes), coverage (rules should cover as many examples as possible) and simplicity (rules should be simple to describe, i.e. use as few attributes as possible). BioHEL uses the minimum description length principle (MDL) [19] to realise this balance. The overall fitness function $F$ is defined in equation 1.

$$
F=T L \times W+E L,
$$

$F$ (where lower is better) is comprised of two terms. The first term is the theory length $(T L)$ which measures the simplicity of each rule. It is multiplied by a $W$ parameter to mitigate its impact on the overall fitness formula. $W$ is automatically adjusted following the heuristic proposed in [2]. The second term, EL, is the exception length, a measure of the quality of the rules that combines its accuracy, Acc, and its coverage, Cov. This is given by:

$$
E L=(1-A c c)+(1-C o v) .
$$

Accuracy is measured by the proportion of training instances the rule classifies correctly to the total number of instances that it matches:

$$
A c c=\frac{\text { correctly classified }}{\text { total matched }} .
$$

Coverage is classically defined as the percentage of examples from the training set that is matched by the rule. In BioHEL this concept is refined in two directions: (1) using Recall $(R C)$ rather than coverage and (2) with the concept of Coverage Breakpoint (CB). BioHEL employs the recall to focus only on the examples belonging to the class predicted by the rule. The recall is defined as the percentage of examples from the rule's class that are covered by the rule. Moreover, when datasets are affected by class imbalance, recall is more suitable than coverage. The recall is further filtered by specifying a system-wide parameter, called Coverage Breakpoint (CB), that indicates the rules that are good enough. Intuitively, this means that most of the recall reward, specified by the $C R$ parameter, will be assigned to rules only if they reach at least $C B$ recall. Notice that the $C B$ parameter requires careful tuning across datasets, but it is very effective in complex and noisy datasets.

$$
C o v= \begin{cases}C R \times \frac{R C}{C B}, & R C<C B \\ C R+(1-C R) \times \frac{R C-C B}{1-R C}, & R C \geqslant C B\end{cases}
$$


The theory length $(T L)$ term of the fitness function (equation 1) is designed to favour simple rules.

$$
T L=\frac{\sum_{i=1}^{N_{a}} 1-\operatorname{size}\left(R_{i}\right) / \operatorname{size}\left(D_{i}\right)}{N_{a}},
$$

where $N_{a}$ is the total number of attributes in the dataset, $\operatorname{size}\left(R_{i}\right)$ is the length of the interval (upper bound - lower bound) specified in a rule for attribute $i$, and size $\left(D_{i}\right)$ is the size of the domain for attribute $i$. TL quantifies the volume of the hyper-rectangle defined by the rule, and given that only relevant attributes contribute to the formula, it will promote rules that contains fewer attributes.

\subsection{Studied knowledge representations}

BioHEL's ALKR was re-factored to be able to explicitly specify certain types of predicates. Rather than containing a list of expressed attributes and, for each of these, an associated lower and upper bound, now the representation contains a list of predicates, which can be of four different kinds:

- Hyper-rectangle: Att X $\in[$ Lower, Upper $]$

- Greater than: AttX $>$ Lower

- Less than: AttX $<$ Upper

- Ratio: AttX/AttY > Threshold

The types of predicates are designed to capture different biological phenomena, for example, less and greater than describe down and up regulation respectively. Similar, the ratio aims to represent the interaction between biological entities due to e.g. regulation: when the high/low presence of one e.g. gene activates the expression of another gene.

whose abundance often regulate complex processes such as transcription or regulation.

For the first three predicate types, the range of legal values for lower and upper are determined by the min and max values observed in the training set for a given attribute. For the ratio predicates the threshold can take a value between 1 and 5. Using the presented knowledge representations, a BioHEL's rule contains a set of predicates, which can all belong to the same kind or can be mixed (belong to multiple representations). The proposed representations are illustrated in Figure 1.

In the case of the mixed representation, the initial population of the GA would initialise rules in which all the predicate types have the same chance to appear, the evolutionary process will then identify the appropriate type for the given dataset. This change in knowledge representation impacts BioHEL in several parts of its working cycle:

- Initialisation. When creating initial rules a parameter specifies the expected number of predicates per rule. Once the actual number of predicates for a specific rule is decided, these are randomly created with a single constrain: we explicitly prevent the creation of incompatible predicates in the 


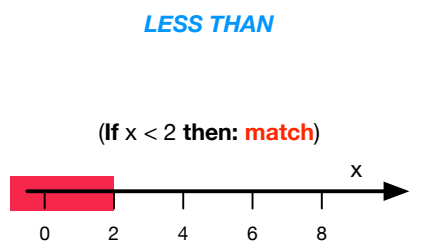

GREATER THAN

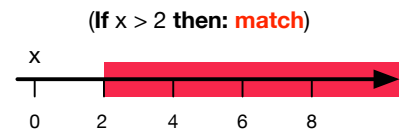

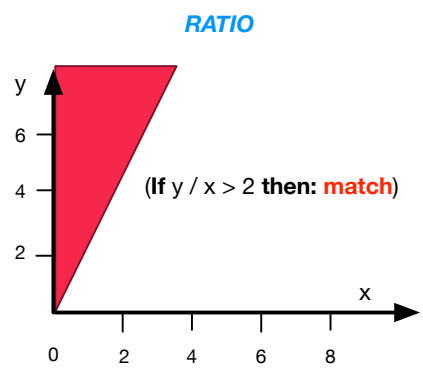

HYPER-RECTANGLE

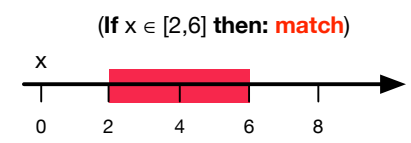

MIX

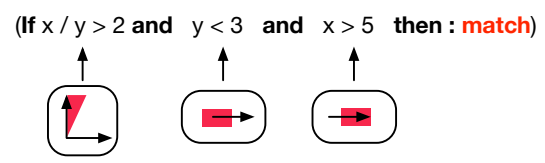

Fig. 1. Illustration of the different types of rule representations studied in this paper

same rule. Two predicates are declared incompatible if (1) they belong to the same type and (2) they share the same attribute(s).

- Crossover. The original 1-point crossover of BioHEL is replaced with a procedure that shares some similarities with the classic uniform crossover: First we iterate over one rule, comparing each of its predicates to the predicates of the second rule. If the two predicates are incompatible then they are copied to different offspring rules, randomly deciding which offspring takes each of them. If a predicate is compatible with all predicates in the other rule, it is randomly assigned to one of the two offspring. After all the predicates from the first rule are assigned, any predicate from the second that was not assigned by the incompatibility check is then randomly associated to an offspring. All the random choices between offsprings occur with a 50-50 chance.

- Mutation. BioHEL uses an individual-wise mutation probability. Once an individual is selected for mutation, one of its predicates is randomly chosen with uniform probability to be mutated. Depending on the predicate type, the action will be different: in hyper-rect, the lower and upper bounds can be mutated. In greater-than only the lower bound can be mutated. In less than only the upper bound, and in the ratio predicate with $50 \%$ chance either (a) the threshold will be mutated or (b) the attributes in the ratio will be inverted. Please note that the actual attributes associated to the 
predicate do not change in any mutation operation. As in the original ALKR representation it will be the role of the specialise operator to create new predicates with different attributes

- Theory length formula. BioHEL's MDL-based fitness function needs to specify the theory length, an estimation of the complexity of a rule. In the original ALKR representation, the theory length was the inverse of the volume of the hyper-rectangle specified by a rule. This definition is maintained for all predicate types, and it is represented by the red area in Figure 1.

- Data normalisation. The original BioHEL's representation did not require any data normalisation. However, the ratio predicate can generate strange behaviours if some of the attributes present negative values. Therefore, all datasets are normalised so variables present only values between 0 and 1

\section{$2.3 \quad$ FuNeL}

FuNeL [14] is a recently proposed method to infer functional networks from rule-based machine learning models. As mentioned in the introduction, FuNeL applies the co-prediction principle: It infers that two biological elements (e.g. genes, in reality attributes of a dataset) need to be connected in the network if they appear together in the rules generated by BioHEL. The way in which we have used FuNeL in this paper has two stages:

1. Rule-based network generation BioHEL is run 1000 times on the same data to generate 1000 different rule sets. From the analysis of the generated rule-sets: (1) all the pairs of attributes co-occurring (together) in the same rules are identified and (2) is counted the number of times that an attribute, overall, is used in the rules (called node score).

2. Permutation test Sometimes, spurious edges might appear in the network. To prevent this situation, FuNeL employs a permutation test: a copy of the dataset (permutation) is generated by scrambling the class labels across samples. Then, BioHEL is applied to the permuted data, and the (random) node score is computed. The permutation test is performed using the node score rather than the edge score (number of times two attributes appear together in the same rule) because, when dealing with high dimensionality datasets such as the one employed for this work, the latter do not contain a signal strong enough to filter out the spurious elements: Even strong edges would appear very few times across rule sets and hence would be difficult to differentiate from weak edges.. By repeating this permutation process 100 times, a distribution of (random) node scores is generated for each attribute. Then, using a one-tailed permutation test, FuNeL assigns to each node a pvalue that represents the likelihood to draw its node score by chance (from the distribution of the permutated node scores). Th final FuNeL network contains the nodes with a p-value $<0.05$ with their direct neighbours. 


\begin{tabular}{|l|c|c|c|}
\hline Dataset & \#Instances & \#Attributes & Source \\
\hline AML & 54 & 12625 & {$[26]$} \\
Breast/Colon cancer & 52 & 22283 & {$[8]$} \\
CNS embryonal tumour & 60 & 7129 & {$[18]$} \\
Diffuse large B-cell lymphoma & 77 & 2647 & {$[20]$} \\
Leukemia & 72 & 7129 & {$[11]$} \\
Lung (Harvard) & 181 & 12534 & {$[12]$} \\
Lung (Michigan) & 96 & 7129 & {$[7]$} \\
Prostate & 102 & 12600 & {$[21]$} \\
\hline
\end{tabular}

Table 1. Eight microarray datasets which are publicly available and their sources.

\begin{tabular}{|l|l|}
\hline Parameter & Value \\
\hline GA Iterations & 50 \\
Crossover probability & 0.6 \\
Selection algorithm & tournament \\
Tournament size & 4 \\
Population size & 500 \\
Individual wise mutation probability & 0.6 \\
Repetitions of rule learning process & 2 \\
Iteration of activation & 10 \\
Initial theory length ratio & 0.25 \\
Weight relax factor & 0.90 \\
Coverage ratio & 0.90 \\
Expected number of predicates in initial rules & 15 \\
Probability of generalise & 0.25 \\
Probability of specialise & 0.25 \\
Default class & majority class \\
\hline
\end{tabular}

Table 2. BioHEL configuration

\section{Experimental design}

To test the new BioHEL's knowledge representations we selected eight publicly available cancer-related microarray datasets (see Table 1) as test data to demonstrate the behaviour of the new BioHEL knowledge representations. We tested five different settings for BioHEL: one setting for each predicate type, plus the mixed representation. The settings of BioHEL are represented in Table 3.

Coverage breakpoint calibration As discussed in section 2.1, the behaviour of BioHEL can be very sensitive to the parameters used, particularly the coverage breakpoint. For each type of predicate and dataset, we tested coverage breakpoints $\{0.05,0.10,0.15,0.20\}$ in order to find the value yielding the most accurate classification. To choose the appropriate value for each scenario we employed a 10-fold cross-validation. For these experiments, in which BioHEL is used to make predictions (unlike later in the network generation process), we used an ensemble of 1000 BioHEL rule sets generated from the same training data with 
different random seeds, following [3]. The coverage breakpoint giving the highest cross-validation accuracy was selected for the network generation experiments.

\subsection{Network generation and Analysis}

The network analysis protocol is represented in Figure 2. Dataset by dataset, FuNeL is run using the five different BioHEL knowledge representation settings to generate five different networks. From the resulting networks, we tested the overlap of nodes across knowledge representations. The nodes in the generated networks correspond to the probes of the analysed transcriptomics data. After the network generation, we mapped each probe to the relative gene symbol (HUGO ID). Then, we analysed the Gene Ontology (GO) terms associated with each gene. We used the PANTHER database ([17]) to conduct an enrichment analysis which identifies the GO terms that are common to a statistically significant proportion of the genes (nodes). Finally, we performed a pairwise comparison of the GO terms to assess the knowledge representations that extracted similar biological information.

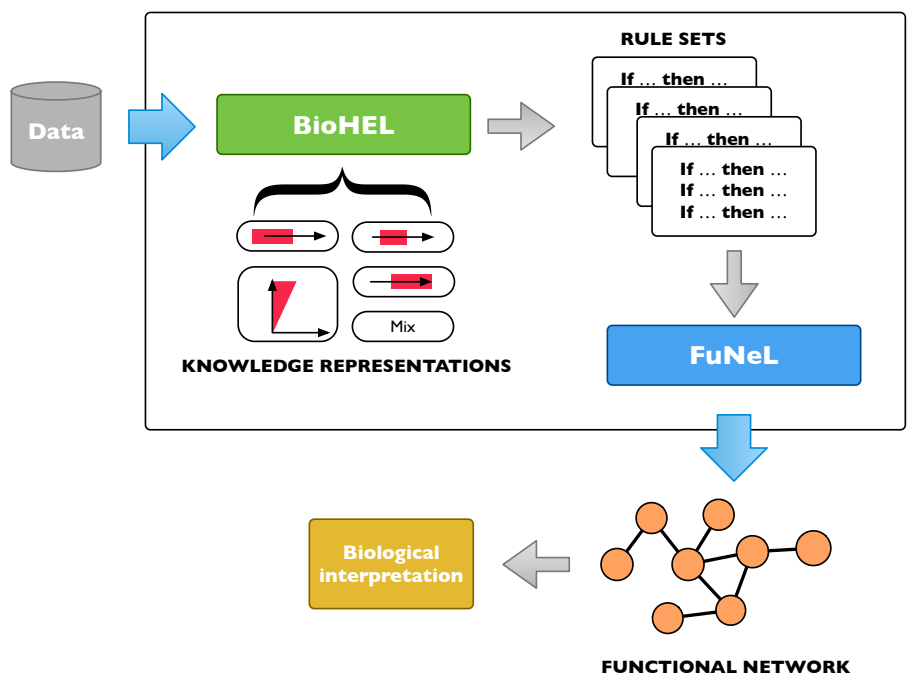

Fig. 2. Representation of the network construction and analysis protocol

\section{Results}

\subsection{Coverage Breakpoint}

In Table 3 we report, for each dataset and knowledge representation, the best coverage breakpoint and the accuracy that BioHEL obtained by using it. These coverage breakpoints were later used in the network generation process. In general, representations that operate with a higher coverage breakpoint are preferred, as this encourages rules which are more general and so counteracts any 


\begin{tabular}{|l|c|c|c|c|c|}
\hline & Hyper-rectangle & Greater than & Less than & Ratio & Mix \\
\hline AML & 0.05 & 0.15 & 0.10 & 0.20 & 0.15 \\
Acc & $\mathbf{6 6 . 7}$ & 64.8 & 61.1 & 65.0 & 64.8 \\
\hline B-C & 0.20 & 0.05 & 0.20 & 0.05 & 0.15 \\
Acc & $\mathbf{9 4 . 2}$ & 91.1 & 86.5 & 92.0 & $\mathbf{9 4 . 2}$ \\
\hline CNS & 0.20 & 0.20 & 0.20 & 0.20 & 0.15 \\
Acc & 70.0 & 50.0 & $\mathbf{7 1 . 7}$ & 65.0 & 70.0 \\
\hline Dlbcl & 0.15 & 0.10 & 0.20 & 0.05 & 0.20 \\
Acc & 53.4 & $\mathbf{6 2 . 0}$ & 46.6 & 53.4 & 60.3 \\
\hline Leukemia & 0.20 & 0.20 & 0.20 & 0.20 & 0.20 \\
Acc & $\mathbf{9 8 . 6}$ & 97.2 & 94.4 & $\mathbf{9 8 . 6}$ & $\mathbf{9 8 . 6}$ \\
\hline LungH & $N P$ & $N P$ & $N P$ & $N P$ & $N P$ \\
Acc & 98.9 & 98.9 & 98.9 & $\mathbf{9 9 . 4}$ & $\mathbf{9 9 . 4}$ \\
\hline LungM & $N P$ & $N P$ & $N P$ & $N P$ & $N P$ \\
Acc & 98.9 & $\mathbf{1 0 0 . 0}$ & $\mathbf{1 0 0 . 0}$ & $\mathbf{1 0 0 . 0}$ & $\mathbf{1 0 0 . 0}$ \\
\hline Prostate & 0.20 & 0.20 & 0.20 & 0.15 & 0.20 \\
Acc & $\mathbf{9 2 . 2}$ & 91.1 & 87.3 & 90.2 & $\mathbf{9 2 . 2}$ \\
\hline \hline Average & 0.175 & 0.163 & 0.188 & 0.156 & 0.181 \\
Acc & 84.1 & 81.9 & 80.8 & 83.0 & $\mathbf{8 4 . 9}$ \\
\hline
\end{tabular}

Table 3. The coverage breakpoint parameters selected after our initial cross validation experiments and the accuracy they obtained. NP denotes that there was no preferred breakpoint parameter. Best accuracy for each dataset is marked in bold

tendency of the representation to over-fit to the specifics of the training data. In average all representations prefer higher $\mathrm{CB}$ values (close to 0.2 , the maximal tested parameter). In some cases, all $\mathrm{CB}$ values gave similar results. Examples are the LungM dataset where all the representations were perfect or near perfect with all the coverage breakpoints. In such cases, the largest coverage breakpoint was used to encourage the rule sets that cover more instances of the data. The mixed representation followed by the hyper-rectangle one gave the best results (obtaining the best accuracy in 5 and 4 out of the 8 datasets, respectively. Mixed is able to tap on all other representations, so it is expected to obtain better results. On the other hand, the hyper-rectangle representation is the less constrained of all predicate types and its flexibility makes it useful for making predictions. The ratio predicates are the third best representation type, and the most constrained ones, greater-than and less-than are the worst performing.

\subsection{Analysis of the FuNeL networks}

The network generation acts as a feature selection mechanism, narrowing the field of thousands of attributes via permutation testing and rule learning to select the subset of attributes that have a biological connection to the classification of the data. Table 4 reports the number of nodes of the networks generated by different knowledge representations with each dataset. It is interesting to note that the ratio knowledge representation selects much fewer attributes from the 


\begin{tabular}{|l|c|c|l|c|c|}
\hline Dataset & KR & Selected & Dataset & KR & Selected \\
\hline AML & HR & 2942 & Leukemia & HR & 1678 \\
(12681 & GT & 3540 & (7129 & GT & $\mathbf{1 4 1 3}$ \\
attributes) $)$ & LT & 3626 & attributes) & LT & 1501 \\
& R & $\mathbf{2 1 3 1}$ & & R & 1452 \\
& M & 4011 & & M & 2040 \\
\hline B-C & HR & 1347 & LungH & HR & $\mathbf{9 5 3}$ \\
(22282 & GT & 1276 & $\mathbf{1 2 5 3 3}$ & GT & 1275 \\
attributes) $)$ & LT & 1258 & attributes) & LT & 2238 \\
& R & $\mathbf{6 9 0}$ & & R & 2804 \\
& M & 1766 & & M & 2512 \\
\hline CNS & HR & 3049 & LungM & HR & $\mathbf{4 4 1}$ \\
(7129 & GT & 3591 & (7129 & GT & 693 \\
attributes) $)$ & LT & 3040 & attributes) & LT & 1160 \\
& R & $\mathbf{2 3 3 9}$ & & R & 1227 \\
& M & 3770 & & M & 919 \\
\hline Dlbcl & HR & 2858 & Prostate & HR & 2991 \\
(7129 & GT & 2919 & (12600 & GT & 3422 \\
attributes) $)$ & LT & 3061 & attributes) & LT & 4159 \\
& R & $\mathbf{1 0 8 3}$ & & R & $\mathbf{2 7 8 6}$ \\
& M & 3415 & & M & 4120 \\
\hline
\end{tabular}

Table 4. Number of nodes in the networks generated by each knowledge representation. Bold marks the knowledge representation generating the smallest network

data (generates the smallest networks, nodes-wise, for 5 out of the 8 datasets), while the mixed representation selects the most attributes.

The next step is to assess the overlap between networks. For each network, we ranked the attributes by node degree, then we counted how often the top 10 nodes of one network were found in the top 50 nodes of the network generated, with a different knowledge representation, from the same dataset.The result of this analysis is shown in Table 5. We see that the hyper-rectangle and mixed predicate types were the highest scorers (showing the most similarity with other knowledge representations), while the other representations scored very differently on different datasets. The ratio predicate type had the lowest overlap in 6 of the 8 datasets, which was to be expected as it operates in a fundamentally dissimilar manner. Low overlap suggests that the ratio type is identifying attributes and features that are not discovered by the other representations. Interestingly it shared most similarity with the greater than representation, even more than with the mixed type. Again, we see that the behaviour of the representations varies greatly from dataset to dataset, but we can also see that once again the mixed representation has very good overlap with all representations using a single predicate type, as does the hyper-rectangle representation.

The final step of the network validation is to quantify the biology captured by such network. We computed the similarity between the sets of GO terms identified by each knowledge representation on a given dataset. We queried the PANTHER database with the lists of genes identified by the probe IDs which 


\begin{tabular}{|c|c|c|c|c|c|c|c|c|c|c|c|}
\hline AML & $\mathrm{H}$ & G & LT & $\mathrm{R}$ & $\mathrm{M}$ & Leukemia & $\mathrm{H}$ & G & LT & $\mathrm{R}$ & M \\
\hline $\mathrm{HR}$ & - & 65 & 56 & 56 & 67 & $\mathrm{HR}$ & - & 54 & 65 & 48 & 59 \\
\hline GT & 65 & - & 56 & 49 & 65 & GT & 54 & - & 52 & 56 & 59 \\
\hline LT & 56 & 56 & - & 46 & 63 & LT & 65 & 52 & - & 46 & 58 \\
\hline$R$ & 56 & 49 & 46 & - & 52 & $\mathrm{R}$ & 48 & 56 & 46 & - & 52 \\
\hline M & 67 & 69 & 63 & 52 & - & M & 59 & 59 & 58 & 52 & - \\
\hline B-C & $\mathrm{H}$ & G & LT & $\mathrm{R}$ & M & Lung & $\mathrm{H}$ & G & LT & R & M \\
\hline $\mathrm{HR}$ & - & 49 & 44 & 44 & 5 & $\mathrm{HR}$ & 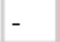 & 53 & 51 & 48 & 50 \\
\hline GT & 49 & - & 33 & 39 & 46 & GT & 53 & - & 48 & 65 & 60 \\
\hline LT & 44 & 33 & - & 30 & 32 & LT & 51 & 48 & - & 53 & 54 \\
\hline $\mathrm{R}$ & 44 & 39 & 30 & - & 32 & $\mathrm{R}$ & 48 & 65 & 53 & - & 6 \\
\hline M & 51 & 46 & 32 & 32 & - & M & 50 & 60 & 54 & 66 & - \\
\hline$\underline{\mathrm{CNS}}$ & $\mathrm{H}$ & G & LT & $R$ & M & Lung & $\mathrm{H}$ & G & LT & $R$ & M \\
\hline $\mathrm{HR}$ & - & 71 & 72 & 64 & 74 & $\mathrm{HR}$ & - & 52 & 34 & 39 & 42 \\
\hline GT & 71 & - & 66 & 62 & 75 & GT & 52 & - & 37 & 54 & 54 \\
\hline LT & 72 & 66 & - & 65 & 71 & LT & 34 & 37 & - & 49 & 49 \\
\hline $\mathrm{R}$ & 64 & 62 & 65 & - & 63 & $\mathrm{R}$ & 39 & 54 & 49 & - & \\
\hline M & 74 & 75 & 71 & 63 & - & $\mathrm{M}$ & 42 & 54 & 49 & 63 & - \\
\hline DLBCL & $\mathrm{H}$ & G & LT & $R$ & M & Prost & $\mathrm{H}$ & $G$ & LT & R & M \\
\hline HR & - & 68 & 73 & 44 & 60 & HR & - & 67 & 72 & 66 & 62 \\
\hline GT & 68 & 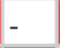 & 67 & 43 & $6 s$ & GT & 67 & - & 63 & 67 & 65 \\
\hline LT & 73 & 67 & - & 43 & 70 & LT & 72 & 63 & - & 63 & 71 \\
\hline $\mathrm{R}$ & 44 & 43 & 43 & - & 39 & $\mathrm{R}$ & 66 & 67 & 63 & - & 66 \\
\hline M & 69 & 69 & 70 & 39 & - & $\mathrm{M}$ & 62 & 65 & 71 & & \\
\hline
\end{tabular}

Fig. 3. Heatmap representing the overlap between the biological annotation captured by different knowledge representations, quantified using the pairwise Jaccard indexes. Colouring is from white to red linearly, scaled between the minimal and maximal values for each dataset. Colour intensity is not comparable across datasets.

formed nodes in each network. PANTHER identified GO terms that were statistically overrepresented among those genes. The Jaccard similarity function is used to quantify such overlap. Figure 3 shows an heatmap generated from the pairwise Jaccard indexes. We can see interesting behaviour from the ratio predicates, which overall has the lowest average overlap with the other representations, but beats all of the other single type representations for both lung cancer datasets. The two lung cancer datasets show very similar profiles despite being collected separately and having different dataset sizes (12534 attributes for the Harvard set and 7129 attributes for the Michigan set). This seems interesting and might suggest a possible particular biology related to lung cancer, however further analysis using other cases of multiple datasets associated with the same disease (e.g. prostate cancer) are necessary to fully address this observation. 


\section{Conclusions}

This paper focuses on analysing the power of extracting knowledge from biological data that evolutionary rule-based machine learning has. Particularly we studied a core component of rule-based machine learning: its knowledge representation. More specifically we analysed how to define the predicates that constitute the rules created by these methods. We have implemented several types of predicates within the BioHEL evolutionary machine learning system and tested them on a set of 8 cancer-related transcriptomics datasets. To quantify the knowledge extraction power of these knowledge representations, we generated functional networks from the rule sets produced by BioHEL and then assessed the overlap between networks generated from the same dataset using different representations. Our results show that different representations generate networks of varying sizes and with relatively low overlap between important nodes. Nevertheless, the overlap between the biological annotation of different networks is much higher. This result leads to a very interesting observation: each representation is able to capture only certain patterns from the data, hence different nodes. However, these patterns are complementary parts of the same story, and this is why similar biological annotation emerged. Different knowledge representations allow us to analyse biological problems from different angles, each one interprets the data in diverse ways and gives us a unique perspective of the phenomena being analysed. Thus, it is crucial not to restrict the biological data analytics process to a reduced/specific type of knowledge representation, because it will only be able to provide partial (and probably biased) knowledge. Moreover, we observed how our results vary across datasets, meaning that different biological problems are better explained by distinct knowledge representation. Therefore, the analytics process needs to be carefully tuned so that we choose the knowledge representation that is more suitable for the range of biological questions that we are planning to address. To achieve this aim a very thorough exercise of characterising knowledge extraction across all kinds of knowledge representations and biological systems (species, tissues, processes, diseases) needs to be performed. In future work, we would also like to extend this comparison to other non-evolutionary methods of inferring biological networks as well as quantifying in much further depth the biological knowledge contained in the generated networks. Furthermore, we will try to test the proposed approach using different type of biological data. In here we employed data generated from a well-established technology (microarray), in future, we will analyse other more modern types such as RNAseq, lipidomics, proteomics etc. Finally, it would also be interesting to assess the impact of the knowledge representations in a different biological knowledge extraction task: biomarker identification [22].

Acknowledgments. This work was supported by the Engineering and Physical Sciences Research Council [EP/N031962/1]. We are grateful to the School of Computing Science of Newcastle University for the access to its High Performance Computing Cluster. We thank the anonymous reviewers for the valuable feedback received. 


\section{References}

1. Bacardit, J., Burke, E.K., Krasnogor, N.: Improving the scalability of rule-based evolutionary learning. Memetic Computing 1, 55-67 (2009)

2. Bacardit, J., Garrell, J.M.: Bloat control and generalization pressure using the minimum description length principle for a pittsburgh approach learning classifier system. In: Learning Classifier Systems, Revised Selected Papers of the International Workshop on Learning Classifier Systems 2003-2005, pp. 59-79. Springer-Verlag, LNCS 4399 (2007)

3. Bacardit, J., Krasnogor, N.: Empirical evaluation of ensemble techniques for a pittsburgh learning classifier system. In: Learning Classifier Systems, Revised Selected Papers of IWLCS 2006-2007. pp. 255-268. LNAI 4998, Springer-Verlag (2008)

4. Bacardit, J., Stout, M., Hirst, J.D., Valencia, A., Smith, R.E., Krasnogor, N.: Automated alphabet reduction for protein datasets. BMC Bioinformatics 10, 6 (2009)

5. Bacardit, J., Widera, P., Márquez-Chamorro, A., Divina, F., Aguilar-Ruiz, J.S., Krasnogor, N.: Contact map prediction using a large-scale ensemble of rule sets and the fusion of multiple predicted structural features. Bioinformatics (2012)

6. Bassel, G.W., Glaab, E., Marquez, J., Holdsworth, M.J., Bacardit, J.: Functional network construction in arabidopsis using rule-based machine learning on largescale data sets. The Plant Cell 23(9), 3101-3116 (Sep 2011)

7. Beer, D.G., Kardia, S.L.R., Huang, C.C., Giordano, T.J., Levin, A.M., Misek, D.E., Lin, L., Chen, G., Gharib, T.G., Thomas, D.G., Lizyness, M.L., Kuick, R., Hayasaka, S., Taylor, J.M.G., Iannettoni, M.D., Orringer, M.B., Hanash, S.: Geneexpression profiles predict survival of patients with lung adenocarcinoma. Nature medicine 8(8) (Aug 2002)

8. Chowdary, D., Lathrop, J., Skelton, J., Curtin, K., Briggs, T., Zhang, Y., Yu, J., Wang, Y., Mazumder, A.: Prognostic gene expression signatures can be measured in tissues collected in rnalater preservative. The Journal of molecular diagnostics : JMD 8(1) (Feb 2006)

9. Fainberg, H.P., Bodley, K., Bacardit, J., Li, D., Wessely, F., Mongan, N.P., Symonds, M.E., Clarke, L., Mostyn, A.: Reduced neonatal mortality in meishan piglets: A role for hepatic fatty acids? PLOS ONE 7(11), 1-9 (11 2012)

10. Glaab, E., Bacardit, J., Garibaldi, J.M., Krasnogor, N.: Using rule-based machine learning for candidate disease gene prioritization and sample classification of cancer gene expression data. PLoS ONE 7(7), e39932 (07 2012)

11. Golub, T.R., Slonim, D.K., Tamayo, P., Huard, C., Gaasenbeek, M., Mesirov, J.P., Coller, H., Loh, M.L., Downing, J.R., Caligiuri, M.A., Bloomfield, C.D., Lander, E.S.: Molecular classification of cancer: Class discovery and class prediction by gene expression monitoring. Science 286(5439), 531-537 (1999)

12. Gordon, G.J., Jensen, R.V., Hsiao, L.L., Gullans, S.R., Blumenstock, J.E., Ramaswamy, S., Richards, W.G., Sugarbaker, D.J., Bueno, R.: Translation of microarray data into clinically relevant cancer diagnostic tests using gene expression ratios in lung cancer and mesothelioma. Cancer research 62(17) (Sep 2002)

13. Hemberg, E., Veeramachaneni, K., Dernoncourt, F., Wagy, M., O'Reilly, U.M.: Efficient training set use for blood pressure prediction in a large scale learning classifier system. In: Proceedings of the 15th Annual Conference Companion on Genetic and Evolutionary Computation. pp. 1267-1274. GECCO '13 Companion, ACM, New York, NY, USA (2013) 
14. Lazzarini, N., Widera, P., Williamson, S., Heer, R., Krasnogor, N., Bacardit, J.: Functional networks inference from rule-based machine learning models. BioData Mining 9(1), 28 (2016)

15. Marcozzi, M., Divina, F., Aguilar-Ruiz, J.S., Vanhoof, W.: A novel probabilistic encoding for eas applied to biclustering of microarray data. In: Proceedings of the 13th Annual Conference on Genetic and Evolutionary Computation. pp. 339-346. GECCO '11, ACM, New York, NY, USA (2011)

16. Martinez-Ballesteros, M., Nepomuceno-Chamorro, I.A., Riquelme, J.C.: Discovering gene association networks by multi-objective evolutionary quantitative association rules. Journal of Computer and System Sciences (2013)

17. Mi, H., Poudel, S., Muruganujan, A., Casagrande, J.T., Thomas, P.D.: Panther version 10: expanded protein families and functions, and analysis tools. Nucleic acids research 44(D1) (Jan 2016)

18. Pomeroy, S.L., Tamayo, P., Gaasenbeek, M., Sturla, L.M., Angelo, M., McLaughlin, M.E., Kim, J.Y.H., Goumnerova, L.C., Black, P.M., Lau, C., Allen, J.C., Zagzag, D., Olson, J.M., Curran, T., Wetmore, C., Biegel, J.A., Poggio, T., Mukherjee, S., Rifkin, R., Califano, A., Stolovitzky, G., Louis, D.N., Mesirov, J.P., Lander, E.S., Golub, T.R.: Prediction of central nervous system embryonal tumour outcome based on gene expression. Nature 415(6870) (Jan 2002)

19. Rissanen, J.: Modeling by shortest data description. Automatica vol. 14, 465-471 (1978)

20. Shipp, M.A., Ross, K.N., Tamayo, P., Weng, A.P., Kutok, J.L., Aguiar, R.C.T., Gaasenbeek, M., Angelo, M., Reich, M., Pinkus, G.S., Ray, T.S., Koval, M.A., Last, K.W., Norton, A., Lister, T.A., Mesirov, J., Neuberg, D.S., Lander, E.S., Aster, J.C., Golub, T.R.: Diffuse large b-cell lymphoma outcome prediction by gene-expression profiling and supervised machine learning. Nature medicine $8(1)$ (Jan 2002)

21. Singh, D., Febbo, P.G., Ross, K., Jackson, D.G., Manola, J., Ladd, C., Tamayo, P., Renshaw, A.A., D’Amico, A.V., Richie, J.P., Lander, E.S., Loda, M., Kantoff, P.W., Golub, T.R., Sellers, W.R.: Gene expression correlates of clinical prostate cancer behavior. Cancer Cell 1(2), 203 - 209 (2002)

22. Swan, A.L., Stekel, D.J., Hodgman, C., Allaway, D., Alqahtani, M.H., Mobasheri, A., Bacardit, J.: A machine learning heuristic to identify biologically relevant and minimal biomarker panels from omics data. BMC Genomics 16(1), S2 (2015)

23. Urbanowicz, R.J., Granizo-Mackenzie, A., Moore, J.H.: An analysis pipeline with statistical and visualization-guided knowledge discovery for michigan-style learning classifier systems. IEEE Comp. Int. Mag. 7(4), 35-45 (2012)

24. Urbanowicz, R.J., Andrew, A.S., Karagas, M.R., Moore, J.H.: Role of genetic heterogeneity and epistasis in bladder cancer susceptibility and outcome: a learning classifier system approach. Journal of the American Medical Informatics Association 20(4), 603612 (2013)

25. Venturini, G.: SIA: A supervised inductive algorithm with genetic search for learning attributes based concepts. In: Brazdil, P.B. (ed.) Machine Learning: ECML-93 - Proc. of the European Conference on Machine Learning, pp. 280-296. SpringerVerlag (1993)

26. Yagi, T., Morimoto, A., Eguchi, M., Hibi, S., Sako, M., Ishii, E., Mizutani, S., Imashuku, S., Ohki, M., Ichikawa, H.: Identification of a gene expression signature associated with pediatric aml prognosis. Blood 102(5), 1849-1856 (2003) 


\begin{tabular}{|c|c|c|c|c|c|c|}
\hline AML & Hyper-rectangle & Greater than & Less than & Ratio & Mix & Mean \\
\hline Hyper-rectangle & - & 1 & 1 & 1 & 2 & 1.25 \\
\hline Greater than & 2 & - & 0 & 2 & 7 & 2.75 \\
\hline Less than & 0 & 0 & - & 0 & 4 & 1 \\
\hline Ratio & 1 & 2 & 0 & - & 1 & 1 \\
\hline Mix & 2 & 3 & 2 & 2 & - & 2.25 \\
\hline B-C & Hyper-rectangle & Greater than & Less than & Ratio & Mix & Mean \\
\hline Hyper-rectangle & - & \begin{tabular}{|c|}
0 \\
\end{tabular} & 5 & \begin{tabular}{|l|}
0 \\
\end{tabular} & 5 & 2.5 \\
\hline Greater than & 1 & - & 0 & 2 & 2 & 1.25 \\
\hline Less than & 9 & 0 & - & 0 & 3 & 3 \\
\hline Ratio & 0 & 1 & 0 & - & 0 & 0.25 \\
\hline Mix & 2 & 1 & 0 & 0 & - & 0.75 \\
\hline CNS & Hyper-rectangle & Greater than & Less than & Ratio & Mix & Mean \\
\hline Hyper-rectangle & - & 1 & 6 & \begin{tabular}{|l|}
0 \\
\end{tabular} & 8 & 3.75 \\
\hline Greater than & 0 & - & 0 & 2 & 0 & 0.5 \\
\hline Less than & 6 & 0 & - & 0 & 7 & 3.25 \\
\hline Ratio & 0 & 2 & 0 & - & 0 & 0.5 \\
\hline Mix & 8 & 0 & 8 & 0 & - & 4 \\
\hline Dlbcl & Hyper-rectangle & Greater than & Less than & Ratio & Mix & Mean \\
\hline Hyper-rectangle & - & \begin{tabular}{|c|}
1 \\
\end{tabular} & 4 & \begin{tabular}{|l|}
0 \\
\end{tabular} & 8 & 3.25 \\
\hline Greater than & 3 & - & 0 & 1 & 3 & 1.75 \\
\hline Less than & 5 & 0 & - & 0 & 4 & 2.25 \\
\hline Ratio & 0 & 1 & 0 & - & 0 & 0.25 \\
\hline Mix & 8 & 4 & 1 & 1 & - & 3.5 \\
\hline Leukemia & Hyper-rectangle & Greater than & Less than & Ratio & Mix & Mean \\
\hline Hyper-rectangle & - & 4 & 6 & 3 & 10 & 5.75 \\
\hline Greater than & 5 & - & 0 & 4 & 6 & 3.75 \\
\hline Less than & 10 & 0 & - & 0 & 8 & 4.5 \\
\hline Ratio & 5 & 4 & 0 & - & 3 & 3 \\
\hline Mix & 9 & 6 & 2 & 3 & - & 5 \\
\hline LungH & Hyper-rectangle & Greater than & Less than & Ratio & Mix & Mean \\
\hline Hyper-rectangle & - & \begin{tabular}{|l|}
9 \\
\end{tabular} & 1 & 5 & 10 & 6.25 \\
\hline Greater than & 8 & - & 0 & 3 & 10 & 5.25 \\
\hline Less than & 1 & 0 & - & 0 & 5 & 1.5 \\
\hline Ratio & 5 & 4 & 0 & - & 5 & 3.5 \\
\hline Mix & 9 & 9 & 1 & 4 & - & 5.75 \\
\hline LungM & Hyper-rectangle & Greater than & Less than & Ratio & Mix & Mean \\
\hline Hyper-rectangle & - & 10 & 0 & 7 & 10 & 6.75 \\
\hline Greater than & 9 & - & 0 & 5 & 8 & 5.5 \\
\hline Less than & 0 & 0 & - & 0 & 0 & 0 \\
\hline Ratio & 8 & 7 & 0 & - & 7 & 5.5 \\
\hline Mix & 8 & 10 & 0 & 6 & - & 6 \\
\hline Prostate & Hyper-rectangle & Greater than & Less than & Ratio & Mix & Mean \\
\hline Hyper-rectangle & - & \begin{tabular}{|c|}
6 \\
\end{tabular} & 4 & 4 & 10 & 6 \\
\hline Greater than & 10 & - & 0 & 6 & 10 & 6.5 \\
\hline Less than & 9 & 0 & - & 0 & 10 & 4.75 \\
\hline Ratio & 4 & 5 & 0 & - & 4 & 3.25 \\
\hline Mix & 9 & 6 & 4 & 4 & - & 5.75 \\
\hline Average & 4.88 & 3.03 & 1.41 & 2.03 & 5.31 & \\
\hline
\end{tabular}

Table 5. Overlap between the top nodes (highest degree) across networks. An average is included with emphasis for the representations that had the highest score. 